(2) Open Access Full Text Article

REVIEW

\title{
Is yoga a suitable treatment for rheumatoid arthritis: current opinion
}

This article was published in the following Dove Press journal:

Open Access Journal of Sports Medicine

7 August 2012

Number of times this article has been viewed

\section{Shirley Telles \\ Nilkamal Singh}

Patanjali Research Foundation, Haridwar, India
Correspondence: Shirley Telles Patanjali Research Foundation, Patanjali Yogpeeth, Haridwar, Uttarakhand 249405, India

Tel +9l 01334244805

Fax +9| 01334244805

Email shirleytelles@gmail.com
Abstract: We reviewed published literature regarding the use of yoga for managing rheumatoid arthritis to determine whether adequate evidence exists to suggest its usefulness as a therapy. A search for previous studies involving yoga and rheumatoid arthritis in PubMed yielded eight reports. These studies reported the benefits of yoga in the physical and mental health of patients with rheumatoid arthritis (RA), suggesting that yoga is a useful add-on therapy for RA patients However, all studies showed limitations with respect to sample size, study design, description and duration of yoga intervention, and assessment tools and statistical methods used. Additionally, the studies did not attempt to understand the mechanisms underlying observed benefits. Hence, evidence suggests a definite role of yoga in RA improvement, reducing pain, improving function, and creating a positive mental state. However, detailed analysis and additional studies are necessary to verify these observations.

Keywords: bibliographic database search, PubMed, rheumatoid arthritis, yoga

\section{Background}

Rheumatoid arthritis (RA) is thought to be an inflammatory, autoimmune disorder resulting in degeneration of the synovial lining in joints, making them swollen, stiff, and less functional. ${ }^{1}$ Previous studies have associated psychological stress with autoimmune disorder onset. Physiological response to experimental stressors was examined in patients with rheumatoid arthritis and systemic lupus erythematosus, and altered immune functioning was observed in patients compared with control subjects. ${ }^{2}$ The observed increase in the number of natural killer cells may have been due to an increase in stress hormones such as catecholamines. Another study found a higher risk for autoimmune diseases $(16.7 \%, 95 \%$ confidence interval: $7.9 \%-29.3 \%)$ in Vietnam combat veterans with post-traumatic stress disorder compared to those without posttraumatic stress disorder. ${ }^{3}$

The practice of yoga involves specific physical postures (asanas), breath regulation (pranayamas), concentration (dharana), and meditation (dhyana). ${ }^{4}$ Previous studies have reported stress reduction after practicing yoga in individuals with mental stress. Benefits were observed in persons experiencing work-related stress, ${ }^{5}$ combat stress, ${ }^{6}$ post-traumatic stress disorder due to natural calamities,,${ }^{7,8}$ and stress caused by war or violence. ${ }^{9}$ These results are indirectly useful for patients with rheumatoid arthritis, as stress has been linked to triggering autoimmune disease and worsening rheumatoid arthritis symptoms. Additionally, the effect of yoga 
on the musculoskeletal system ${ }^{10}$ may also be important for managing symptoms of rheumatoid arthritis.

\section{Review of studies examining the use of yoga for treating rheumatoid arthritis}

An electronic search of the PubMed database was conducted using the following keywords: yoga and rheumatoid arthritis, meditation and rheumatoid arthritis, mindfulness meditation and rheumatoid arthritis, and yogic breathing and rheumatoid arthritis. Eight studies were identified, including 2 randomized controlled studies, 2 non-randomized, matched-group studies, 2 non-randomized studies in which the groups were not matched, and 2 single-group studies. Details of the studies are discussed below.

In one of the randomized controlled studies, ${ }^{11}$ subjects with RA ( $n=63$, both sexes) were randomized into a mindfulness-based stress reduction (MBSR) group $(n=31)$ or a wait list control group $(n=32)$. Patients diagnosed with RA by a physician, age $\geq 18$ years, and not in remission from RA according to criteria established by the American College of Rheumatology were included in the study. Patients with major psychiatric illness, active alcohol or drug dependency, diagnosis of fibromyalgia, inability to attend study sessions, participating in another clinical trial, or scheduled for major surgery were excluded from the study. All participants continued prescribed medications. The Symptom Checklist-90-Revised (SCL-90-R) was used to evaluate depressive symptoms and psychological distress, RA disease status was assessed using the Disease Activity Score in 28 joints (DAS28), $100 \mathrm{~mm}$ visual analog scale for patients' assessment of disease and erythrocyte sedimentation rate, well-being measured using Psychological WellBeing Scales, and state of mindfulness using Mindfulness Attention Awareness Scale (MAAS) at baseline, 2 months, and 6 months after baseline. Participants in the MBSR group participated in an MBSR class for 8 weeks, with participants meeting once weekly for 2.5 hours; subjects also attended a full-day retreat. Three certified MBSR teachers taught the classes. After the 8-week program, participants returned for a 2-month follow-up assessment and attended 3 refresher classes during the following 4 months; final assessments were conducted at 6 months post-baseline. After 2 months, analyses were conducted for 28 participants in the MBSR group and 32 participants in the control group for psychological outcomes and 25 participants in the MBSR group and 28 participants in the control group for RA disease activity. After 6 months, analyses were conducted for 28 participants in the MBSR group and 30 participants in the control group for the psychological outcome and 25 participants in the MBSR group and 29 participants in the control group for RA disease activity. The effect of the intervention, adjusted means, and a priori-defined outcome of adjusted mean change from baseline at 2 and 6 months were estimated in 2 (treatment group) Vs 2 (time) mixed model repeated measures regression analyses, with MBSR and the control condition compared using group contrasts using the mixed procedure in SAS version 9.1. Linear regression models were used to assess the relationship between intervention and outcome within the MBSR group.

Between groups, differences after 2 months were not significant for any outcome measures. After 6 months, psychological distress and well-being showed a significant group $\times$ time interaction $(P<0.05)$. Linear regression models revealed that from baseline to 2 months, each one-day increase in practice was associated with an improvement of depressive symptoms by $-0.03(P<0.05)$.

The study was well-designed, although additional objective measurements could have been added. The authors identified limitations of the study as a small sample size, inadequate attention to the control group, and a floor effect. The improvement observed in the MBSR group may not have been due to the effect of intervention but rather other factors, such as group interaction, which was lacking in the control group. Additionally, all participants with a history of depression were assigned to the control group, which may have affected the results. Effect sizes were not calculated.

In another randomized controlled study, ${ }^{12} 144$ patients were clustered into three groups including 6-10 participants and randomly assigned to a mindfulness-based emotion regulation therapeutic program $(M)(n=51)$, cognitive behavioral therapy for pain $(P)(n=47)$, and an education-only group $(E)$ $(\mathrm{n}=44)$. One subject each from the $\mathrm{M}$ and $\mathrm{P}$ groups dropped out of the study before beginning intervention. Participants who were not taking estrogen replacement therapies, did not have lupus, described themselves as having RA at screening and could obtain written confirmation of RA from a rheumatologist were included in the study. All participants were screened for history of depression using mood disorders modules of the Structured Clinical Interview for DSM-IV (SCID-I). Subjects were further stratified into those with recurrent depression $(\mathrm{RD}+)$ and those without recurrent depression (RD-). Assessment of daily pain level, positive and negative affect, depressive symptoms, coping efficacy for pain, pain catastrophizing, and pain control were evaluated using a 30-page diary questionnaire. Participants were 
instructed to fill out the diary half an hour before bedtime each day. Laboratory assessments were conducted for half of the participants to evaluate joint swelling and tenderness by a rheumatologist who palpated 28 joints taken from the Disease Activity Score-28 (DAS-28) and production of interleukin-6 (IL-6) using stimulated IL-6-production assays. The treatment period was 8-weeks and involved a 2-hour weekly session. Interventions were administered in groups of 5-8 participants. The education only group was blind to alternative treatment conditions and the study hypothesis. At 6 months post baseline, the $M$ group included 47 participants, the $\mathrm{P}$ group included 44 participants, and the $\mathrm{E}$ group included 40 participants. Primary data analysis was conducted using multilevel modeling. Model specifications followed Singer's recommendations to identify the best fit model of variance and covariance of the variables being examined. Daily pain scores after intervention were significantly lower than values before intervention $(P<0.001)$. No time $\times$ group interaction or time $\times$ group $\times$ RD interaction was observed. An increase in positive affect was observed for all participants as a function of time $(P<0.001)$. A time $\times$ group interaction showed a significant increase $(P<0.001)$ in positive affect in both the $\mathrm{M}$ and $\mathrm{P}$ groups compared to the $\mathrm{E}$ group. Participants with $\mathrm{RD}+$ in the $\mathrm{M}$ group showed a greater increase $(P<0.001)$ in positive affect than participants in other groups. A significant triple interaction (with RD as a continuous variable) was observed for negative affect, and $\mathrm{RD}+$ participants in the $\mathrm{M}$ group reported a greater decrease $(P<0.01)$ in negative affect compared to participants in other treatment groups. Daily depression symptoms were decreased $(P<0.02)$ post-treatment compared to before treatment. An increase in coping efficacy for pain was observed for all participants $(P<0.001)$; those in the $\mathrm{M}$ and $\mathrm{P}$ groups showed greater improvement $(P<0.01)$ than those in the E group. Participants with $\mathrm{RD}+$ in the $\mathrm{M}$ group showed greater improvement in coping efficacy for pain than those in other groups $(P<0.001)$. Significant time $\times$ group interaction $(P<0.001)$ and time $\times$ group $\times$ RD interactions $(P<0.001)$ were observed, revealing greater benefits of $M$ treatment over other treatments. A significant time effect was observed for pain control $(P<0.01)$; the $\mathrm{P}$ and $\mathrm{E}$ groups showed greater improvement than the $\mathrm{M}$ group.

Physicians rating of joint tenderness showed a significant decrease in tenderness after compared to before treatment $(P<0.001)$ and a significant group $\times$ time interaction $(P<0.05)$. The group $\times$ time $\times \mathrm{RD}$ interaction revealed that $\mathrm{RD}+$ participants in the $\mathrm{M}$ group experienced a greater reduction in tenderness compared to other groups $(P<0.001)$.
Similar results were observed in the analysis of joint swelling. IL-6 production decreased after compared to before treatment in the $\mathrm{P}$ group alone $(P<0.02)$. Analysis of the moderating effects of at least one episode of clinical depression showed a primary effect of depression on IL-6 scores $(P<0.001)$ and a depression $\times$ time interaction $(P<0.001)$, indicating higher scores for depressed participants that were sustained from before to after treatment, but which had no moderating influence on intervention effects. Effect sizes were calculated using Cohen's $d$ coefficient for pain $(d=0.27)$, positive affect $(d=0.78)$, negative affect $(d=-0.89)$, depressive symptoms $(d=0.16)$, coping efficacy $(d=0.65)$, pain catastrophizing $(d=-0.18)$, pain control $(d=-0.44)$, swelling, $(d=-0.62)$, tenderness $(d=-0.83)$, and IL-6 $(d=0.21)$.

The study provided useful information regarding the usefulness of both cognitive behavioral therapy and a mindfulness-based emotion regulation therapeutic program for patients with RA. An impact of interventions on patients with or without history of recurrent depression (RD) was clearly demonstrated in this study. However, there were some limitations to the study. Participants were not screened for RA using standard screening methods, and the method in which participants were randomized to groups was not described. Additionally, the mindfulness intervention used in the study was not the standard mindfulness-based stress reduction (MBSR) technique, but a shorter version of MBSR. The authors did not describe a standardization procedure/ protocol for developing the mindfulness intervention used in the study, and it is not mentioned whether the instructor providing mindfulness meditation was trained in the technique; these differences in intervention may be why no change was observed in IL-6 level in the mindfulness intervention group, which disagrees with the results of other studies. Additionally, most factors (eg, the daily pain level, positive and negative affect, depressive symptoms, coping efficacy for pain, pain catastrophizing and pain control) were measured using unreliable methods. The limitations of the study as described by the authors included a small sample restricted to those with lower income compared to the community.

In a matched paired controlled study, 20 volunteers with RA were allocated to yoga $(n=10)$ and control $(n=10)$ groups. ${ }^{13}$ Both groups continued with prescribed medical treatments without modification. Standard measures such as ring size, duration of morning stiffness, grip strength, Stanford Health Assessment Questionnaire Disability Index (HAQ), and psychological assessment using the General Health Questionnaire (GHQ) were performed at baseline and after 3 months by qualified nurses who were unaware 
of the hypothesis and which groups the participants were in. Participants in the yoga group took part in 2-hour sessions every day for 5 days per week for 3 weeks followed by weekly 2 -hour sessions for an additional 3 months. On days on which sessions were not held, participants practiced for 10-30 minutes at home. The yoga program included gentle physical movements and postures (asanas), breathing techniques (pranayamas), meditation, and lectures on yoga philosophy. Ten patients in the yoga group completed the first 3 weeks but four subjects subsequently dropped out of the study. All participants completed assessments at the end of the trial except for one participant in the yoga group. Greater improvement was observed in left hand grip strength in the yoga group compared to the control group $(P<0.02)$. The study was one of the earliest conducted to determine the effect of yoga in patients with RA. The results regarding improvement in grip strength are encouraging. The limitations mentioned described by the authors included the small sample size and that the duration of intervention was too short. Other limitations of the study were not using a standard screening tool for screening patients with RA, duration of RA in the control group was much longer than in the control group, which may have influenced the study outcome, and the method/s employed to analyze the data were not described.

Another matched paired controlled study examining 40 patients with RA who were divided into a yoga group ( $\mathrm{n}=20,10$ males) and a control group $(\mathrm{n}=20,10$ males $)$ also showed significant improvement in hand grip strength in the yoga group. ${ }^{14}$ No other assessments relevant to RA were carried out; hence, this study did not contribute significantly to understanding the role of yoga in RA.

Forty-seven patients with RA with similar baseline demographics were non-randomly assigned to a yoga group $(n=26)$ and a control group $(n=21) .{ }^{15}$ Patients aged over 18 years, those with a diagnosis fulfilling the criteria of RA based on the American College of Rheumatology (ACR), those willing to give informed consent, and those without physical disability preventing them from participating in yoga practice were included in the study. Participants were assessed using the Health Assessment Questionnaire (HAQ), SF-36 quality of life (SF QOL), visual analog scales relating to pain, global assessment, and fatigue indices, use of disease-modifying anti-rheumatic drugs (DMARD), disease duration, demographics, disease activity scores using 28 joint count (DAS28) and erythrocyte sedimentation rate (ESR) at baseline and at follow-up visit after 12 sessions of yoga. The yoga program duration was 6 weeks, consisting of 2 sessions per week of 1 hour each. The yoga class was conducted by a licensed practitioner with post-graduate qualification in Yoga and Ayurveda. The program included stretches, strengthening, meditation, and deep breathing. Controls were provided theoretical information regarding yoga and RA support group. Data were analyzed using Wilcoxon matched-pairs signed-ranks test. Analyses were carried out on an intent-to-treat basis, with all available data included, regardless of compliance with the protocol. Significant improvements in tender joint count $(P<0.05)$, swollen joint count $(P<0.01)$, DAS $28(P<0.05)$, and HAQ $(P<0.01)$ were observed in the yoga group. No significant change was observed in the control group. The authors have described limitations of the study as not being able to blind the rheumatologist to interventions, lack of group exercise, interaction in the control group, and small sample size. Additionally, participants with diseases other than RA were not excluded from the study and participants were not randomly allocated to yoga and control groups. Effect sizes were not calculated in the study.

Twenty postmenopausal women (ages between 45 and 75 years) were allocated to yoga $(n=11)$ or control $(n=9)$ groups based on the time schedule that was convenient for them. ${ }^{16}$ Patients with active RA based on the American College of Rheumatology (ACR) 1987 revised criteria and in functional class I, II, or III as defined by ACR classification of functional status, receiving concurrent stable doses of diseasemodifying antirheumatic drugs (DMARDs) for a minimum of 4 weeks before the start of the trial, not taking more than $7.5 \mathrm{mg}$ /day of glucocorticoids, who were able to get down to the floor and back up with no more than minimal assistance, and those naïve to yoga were included in the study. Individuals with any other major inflammatory disease, diabetes mellitus, or systemic disease, such as chronic obstructive pulmonary disease, congestive heart failure, stroke, chronic liver failure, or renal disease, those who had joint replacement surgery within the past two months, smokers, and those with a history of drug or alcohol abuse were excluded from the study. Further, participants were not allowed to use narcotic analgesics, except for limited doses of hydrocodone, codeine, or prophoxyphene during the study. Assessments were conducted before and after the 10-week yoga intervention.

The health assessment questionnaire (HAQ) was used to measure disability for individuals with RA, the Beck depression inventory for characteristic attitudes and symptoms of depression, the Berg balance test (BBT) for screening elderly subjects who were at risk for falling, diurnal cortisol variability using a coated-tube radioimmunoassay, 
and resting heart rate using a heart rate monitor. The yoga sessions were conducted over a 10-week interval with 3 classes per week with each class lasting for 75 minutes. The yoga instructor was a certified yoga therapist. Baseline and post-yoga treatment data were compared using two-factor analyses of variance (group $\times$ time) using repeated measures for time. Apart from diurnal cortisol, cortisol awakening response was calculated separately by analyzing the first four morning samples. Diurnal cortisol and awakening responses were also quantified based on overall response, correcting for baseline, or the integrated area under the response curve (AURC). Post-hoc analyses using paired sample $t$-tests were conducted for some instances. The partial eta squared (partial $\eta^{2}$ ) was calculated to determine effect size for group differences lacking statistical significance. In both groups, 2 participants dropped out of the study. Health assessment scores were significantly reduced $(P<0.01)$ in the yoga group and increased $(P<0.01)$ in the control group. Pain significantly decreased $(P<0.05)$ in the yoga group. Berg balance test scores also increased significantly after yoga treatment $(P<0.01)$. An improvement in depression symptoms was observed after yoga $(P<0.05)$. A significant cortisol awakening response $(P<0.01)$ and a significant daytime decrease in cortisol $(P<0.05)$ was observed for both groups. The results of the study indicate the benefits of yoga in patients with RA. The authors mention that lack of randomization to yoga and control groups may have led to self-selection bias. Additionally, not using traditional measures of disease activity, such as the erythrocyte sedimentation rate and evaluating the number of tender and swollen joints (where level of swelling or tenderness were not based on standard criteria), were mentioned as limitations of the study.

A single group study 64 patients with RA (47 females) aged between 20 and 70 years and meeting the criteria of the American College of Rheumatology were included as participants. ${ }^{17}$ All participants were on medication which was not modified during the study. Assessments were conducted using the Stanford Health Assessment Questionnaire (HAQ), hydraulic hand grip dynamometer for hand grip, and laboratory tests for serum rheumatoid factor and C-reactive protein levels (immunoturbidometry) at the beginning and end of one week of intervention. Two yoga sessions were held each day and each session lasted for 2:30 hours. The yoga program included loosening exercises, yoga postures, and breathing techniques. Before and after data were compared using the $t$-test for paired data. Data were analyzed for the entire group and gender wise comparisons were conducted separately.
A significant decrease was observed in the disability index $(P<0.01)$ and rheumatoid factor $(P<0.05)$ of the entire group after intervention. For female participants, a significant improvement was observed in $(P<0.001)$ in dressing, arising, and walking. Similarly, for male participants, a significant improvement was observed in dressing $(P<0.05)$, arising $(P<0.001)$, walking $(P<0.05)$, and grip strength $(P<0.001)$. This study suggested that brief, intensive yoga intervention can improve function and produce serological changes suggestive of immune system changes. However, there were several limitations to the study, including the lack of a control group and a self-selected group which may have influenced results regarding how feelings and beliefs about intervention can influence our behavioral responses. Though this was a residential program, diet was not controlled. Additionally, participant ages varied widely (20 to 70 years). An age-wise analysis of disability index data would provide more useful information regarding observed improvements.

Eight participants with RA ranging in age between 18 and 35 years were enrolled in a study. ${ }^{18}$ They were screened for RA according to the revised 1987 American College of Rheumatology (ACR) criteria. Patients using diseasemodifying antirheumatic medication (stable doses for eight weeks), using non-steroidal anti-inflammatory drugs, using low-dose corticosteroids (doses stable for four weeks), who were willing to provide written informed consent and able to speak and understand English were included in the study. Assessments were conducted before and after intervention using the Pain Disability Index (PDI) to measure pain and functioning, the Health Assessment Questionnaire (HAQ) for arthritis-specific disability, the Short Form-36 (SF-36) for functional health and well-being, the Brief Symptom Inventory (BSI-18) for psychospiritual functioning, the Arthritis Self-Efficacy scale for arthritis patients' beliefs that they could perform specific tasks or behaviors to cope with the consequences of chronic arthritis, the chronic pain acceptance questionnaire to determine the degree to which patients are willing to accept and move on with life despite pain, the 15-item Mindfulness Attention Awareness scale to measure attention to what was occurring at the present moment, semistructured interviews for topics such as perceptions of the impact of the yoga program on functioning including any changes in pain, symptoms, or mood, whether the program met participants' expectations, social effects, what was/was not helpful, whether the intervention was suitable for RA, and a weekly monitoring form to assess patients' weekly pain, anxiety, depression, and energy ratings using a numerical rating scale where 0 denoted no pain and 10 denoted the worst 
Table I Levels of evidence for randomized controlled trials (based on Oxford Center for Evidence-based Medicine)

\begin{tabular}{ll}
\hline Evidence level & Study design \\
\hline $\mathrm{I}$ & High quality RCTs with narrow \\
& confidence intervals \\
2 & Low quality RCTs or high quality cohort \\
& studies \\
3 & Case-control studies \\
4 & Case series or poor case-control studies \\
& or poor cohort studies or case reports \\
\hline
\end{tabular}

Notes: High quality RCTs are those with narrow confidence intervals and $>80 \%$ follow-up rate; Low quality RCTs are those with wide confidence intervals, $<80 \%$ follow-up rate. The Center for Evidence based Medicine defines low-quality cohort studies as "those which have not clearly defined comparison groups and/or failed to measure exposure and outcomes in the same (preferably blinded), objective way in both exposed and non-exposed individuals and/or failed to identify or appropriately control known confounders and/or failed to carry out a sufficiently long and complete follow-up of patients."

Abbreviation: RCTs, randomized controlled trials.

pain imaginable. A weekly monitoring form was provided before intervention, weekly during intervention, and at twomonth follow-up. Participants were also asked to report any adverse events during the classes, any changes in medication, home practice of yoga, and level of physical activity. Participants were taught Iyenger Yoga (IY) for 6 weeks, with two sessions per week each lasting 1.5 hours. An additional class per week was made available for participants who could not attend the regular session. Wilcoxon tests for dependent samples were used to analyze quantitative data and ethnographic content analysis was used to analyze qualitative data for participants who completed the study $(n=5)$. During the first week of the study, 3 participants dropped out of the study. The remaining participants reported significantly less pain, pain disability (impact of pain on ability to participate in basic life activities), and depression after completing the IY program $(P<0.05)$. Effect sizes of PDI $(d=0.12)$, HAQ (disability, $d=0.46$, pain, $d=0.80$ ), SF-36 (physical functioning, $d=-0.39$, vitality, $d=-1.21$, mental health, $d=-0.94$ ), BSI (depression, $d=1.01$, anxiety, $d=0.98$ ), self-efficacy $(d=-0.86)$, chronic pain acceptance $(d=-0.54)$, and mindfulness $(d=-0.74)$ were reported. No statistical tests were conducted for data acquired using the weekly monitoring form. The authors described the limitations of the study as a small sample size and lack of a control group. Additionally, it is possible that too many questionnaires and no objective measurements were used. Hence, the results may have been influenced by the intentions and beliefs of the participants.

\section{Conclusion and summary}

The eight studies that used yoga as an intervention for RA were evaluated according to 'Levels of evidence for randomized controlled trials (based on the Oxford Center for Evidence-based Medicine),' which are summarized in Table 1. ${ }^{19}$ Three studies showed Evidence level 2, ${ }^{11-13}$ four studies showed Evidence level 3, ${ }^{15-18}$ and one study showed Evidence level $4 .{ }^{14}$

After reviewing the eight studies, the following points emerged: (i) each study indicated that yoga is a useful add-on therapy for treating RA, (ii) the studies all had limiting factors, (iii) the most significant benefits were observed when yoga was practiced in combination of physical postures, regulated breathing, meditation, and yoga philosophy, and (iv) there have been no noteworthy attempts to explain the mechanisms underlying the changes observed. In summary, sufficient evidence exists to suggest that yoga should be considered an add-on therapy for rheumatoid arthritis, but further research is necessary to account for the limitations cited above.

\section{Disclosure}

The authors report no conflicts of interest in this work.

\section{References}

1. Poehlmann KM. Rheumatoid Arthritis: The Infection Connection (Targeting and Treating the Cause of Chronic Illness), 2nd ed. Torrance, CA: Satori Press; 2011.

2. de Brouwer SJM, Kraaimaat FW, Sweep FCGJ, et al. Experimental stress in inflammatory rheumatic diseases: a review of psychophysiological stress responses. Arthritis Res Ther. 2010;12(3):R89.

3. Boscarino JA. Posttraumatic stress disorder and physical illness: Results from clinical and epidemiological studies. Ann N Y Acad Sci. 2004;1032:141-153.

4. Taimini IK. The Science of Yoga. Madras, India: The Theosophical Publishing House; 1986.

5. Wolever RQ, Bobinet KJ, McCabe K, et al. Effective and viable mindbody stress reduction in the workplace: a randomized controlled trial. J Occup Health Psychol. 2012;17(2):246-258.

6. Stoller CC, Greuel JH, Cimini LS, Fowler MS, Koomar JA. Effects of sensory-enhanced yoga on symptoms of combat stress in deployed military personnel. Am J Occup Ther. 2012;66(1):59-68.

7. Telles S, Singh N, Joshi M, Balkrishna A. Post traumatic stress symptoms and heart rate variability in Bihar flood survivors following yoga: a randomized controlled study. BMC Psychiatry. 2010;10:18.

8. Telles S, Naveen KV, Dash M. Yoga reduces symptoms of distress in tsunami survivors in the andaman islands. Evid Based Complement Alternat Med. 2007;4(3):503-509.

9. Ehud M, An BD, Avshalom S. Here and now: Yoga in Israeli schools. Int J Yoga. 2010;3(2):42-47.

10. Gonçalves LC, Vale RG, Barata NJ, Varejão RV, Dantas EH. Flexibility, functional autonomy and quality of life (QoL) in elderly yoga practitioners. Arch Gerontol Geriatr. 2011;53(2):158-162.

11. Pradhan EK, Baumgarten M, Langenberg P, et al. Effect of Mindfulness-Based Stress Reduction in rheumatoid arthritis patients. Arthritis Rheum. 2007;57(7):1134-1142.

12. Zautra AJ, Davis MC, Reich JW, et al. Comparison of cognitive behavioral and mindfulness meditation interventions on adaptation to rheumatoid arthritis for patients with and without history of recurrent depression. J Consult Clin Psychol. 2008;76(3):408-421.

13. Haslock I, Monro R, Nagarathna R, Nagendra HR, Raghuram NV. Measuring the effects of yoga in rheumatoid arthritis. BrJ Rheumatol. 1994;33(8):787-788. 
14. Dash M, Telles S. Improvement in hand grip strength in normal volunteers and rheumatoid arthritis patients following yoga training. Indian J Physiol Pharmacol. 2001;45(3):355-360.

15. Badsha H, Chhabra V, Leibman C, Mofti A, Kong KO. The benefits of yoga for rheumatoid arthritis: results of a preliminary, structured 8-week program. Rheumatol Int. 2009;29(12):1417-1421.

16. Bosch PR, Traustadóttir T, Howard P, Matt KS. Functional and physiological effects of yoga in women with rheumatoid arthritis: a pilot study. Altern Ther Health Med. 2009;15(4):24-31.
17. Telles S, Naveen KV, Gaur V, Balkrishna A. Effect of one week of yoga on function and severity in rheumatoid arthritis. BMC Res Notes. 2011;4:118.

18. Evans S, Moieni M, Taub R, et al. Iyengar yoga for young adults with rheumatoid arthritis: results from a mixed-methods pilot study. J Pain Symptom Manage. 2010;39(5):904-913.

19. Phillips B, Ball C, Sackett D, et al. Oxford Centre for Evidence-Based Medicine - levels of evidence. 2009. [cited 2012]; Available from: http://www.cebm.net. Accessed May 18, 2012.

Open Access Journal of Sports Medicine

\section{Publish your work in this journal}

Open Access Journal of Sports Medicine is an international, peer-reviewed, open access journal publishing original research, reports, reviews and commentaries on all areas of sports medicine. The manuscript management system is completely online and includes a very quick and fair peer-review system.
Visit http://www.dovepress.com/testimonials.php to read real quotes from published authors. 